\author{
The Research of a Hail Risk Evaluation under Imperfect Information \\ Wang wei ${ }^{1,2}$, Zhou chao' ${ }^{2}$ Du xin ${ }^{2}$ \\ ${ }^{1}$ Tianjin Meteorology Science Institute, Tianjin 300074, China \\ ${ }^{2}$ College of Environmental Science and Engineering, Nankai University, Tianjin 300071, China
}

\begin{abstract}
Based on the situation of the global warming, hail disasters will maybe cause the higher risk of loss in the future. The author established a risk evaluation model of hail disasters in terms of the combination of probabilistic and physical methods. Moreover, a nonlinear finite element analysis software (ANSYS/LS-DYNA) is utilized to simulate the case that hails dash on the steel body and glass material, whose result was one of the parameters of the evaluation model. Finally, the article carried out a simulation using the hail risk evaluation model and the Monte Carlo random simulation method in terms of the historical statistical data in Tianjin. The result shows that this model can effectively assess hail disaster risk under the situation of insufficient data and information on hail disasters.
\end{abstract}

Keywords: Hail disasters, Risk evaluation, Model, Numerical simulation

\title{
冰雹灾害的风险评估方法的建立与风险模拟研究 ${ }^{*}$ 王 炜 ${ }^{1,2}$ 周超 $^{2}$ 杜金金 $^{2}$ \\ 1 天津市气象科学研究所, 天津, 300074 \\ 2 南开大学环境与工程学院, 天津, 300071
}

摘要: 在全球气候变暖日益加剧的情况下, 未来的冰霄灾害造成的各种经济损失风险可能 会更加严重。针对社会经济发展中可能遇到的冰雹灾害的损失风险问题，作者尝试利用概率方法 和物理方法相结合的手段，建立了相应的冰雹灾害损失的评估模型。并且，利用非线性有限元分 析软件 ANSYS/LS-DYNA 对冰雹撞击钢板和玻璃材料进行了数值模拟, 使用模拟结果确定冰雹 风险评估模型的损失函数参数。最后, 利用天津市的历史统计资料, 将蒙特卡罗随机模拟手段和 冰雹风险评估模型相结合, 模拟分析了天津地区冰雹灾害的损失风险。分析结果表明, 在资料信 息不足的情况下, 建立的冰雹灾害风险分析模型可以有效地评估冰䨙灾害造成的损失。

关键词: 冰雹灾害, 风险评估, 模型, 数值模拟

\section{1. 引言}

冰雹是重要的自然灾害之一。冰雹灾害每 年都给农业、建筑、通讯、电力、交通以及人 民生命财产带来巨大损失。因此, 冰雱灾害的 成因和损失情况受到了许多学者的关注 ${ }^{[1-8]}$ 。 据有关资料统计, 中国每年因冰雱所造成的经 济损失达几亿元甚至几十亿元。例如, 内蒙古 历史年均霨灾造成牲畜伤亡 2818 头, 年均造 成人员伤亡 28 人。最严重的一次是在 1966 年 7 月 23 日, 鄂尔多斯市鄂托克旗, 冰雹砸死 2204 头牲畜, 砸伤 25945 头牲畜 ${ }^{[9]}$ 。2006 年 4 月 13-14 日, 在美国中西部地区发生的一场冰 雨, 造成了大约 18.22 亿美元的损失 ${ }^{[10]}$ 。

在汽车迅速发展的时代, 冰雹对汽车的损 害风险也越来越严重。如 2008 年 6 月 22 日, 一场冰雹袭击了德国北部的一个汽车厂。降落
的冰雨导致大约 30,000 新汽车被损害, 经济 损失达到 1 亿欧元 ${ }^{[11]}$ 。2010 年 5 月 2 日下午, 中国浙江宁波突降冰霄, 降雹时间持续十几分 钟。冰雹过去, 部分汽车的引擎盖上被砸出了 一个个小坑, 还有不少车辆的挡风玻璃都被砸 坏, 更有甚者天窗都被冰雱打碎。

在当前气候变暖的形势下, 极端气候条件 下的冰雹灾害风险会更加严重。但是, 以前的 科学工作者对冰雹灾害的研究主要集中在冰 霖灾害的成灾机理和冰䨌天气预测等方面。由 于对于冰霄灾害的潜在危害认识不足, 以前对 于冰雹灾害的风险评估方法研究的研究工作 开展较少。因此, 针对冰雹灾害的暴露性和承 灾体的脆弱性等对象, 开展定量评估冰霄灾害 的风险的研究工作, 对有效地减少冰霖造成的 经济损失具有实际意义。

*本文得到中国气象局 2010 年软科学重点项目资助（项目编号 2010007）

作者简介: 王炜 (1965-), 男 (汉族), 内蒙古巴彦淖尔盟人, 博士, 副教授, 主要从事风险分析和大气环境数值模拟。 e-mail: wwei356@nankai.edu.cn. 


\section{2. 冰霄灾害的风险识别}

2.1 冰雨灾害的风险成因和风险因素识别

\section{1 .1 冰雨灾害的风险成因和致灾因子} 冰雹是强对流性天气引起的一种剧烈的 气象灾害。一般情况下, 冰䨚灾害发生的范围 较小、时间较短, 但是它由于具有发生突然、 损失强度大的特性, 成为严重气象灾害之一。

冰䨌灾害的主要风险源是雷暴云中降落 的冰雹颗粒。所以冰雱灾害的风险大小同冰雨 的粒径大小有关。然而, 冰雹的粒径大小又同 冰雱的成雱机制密切相关。因为, 冰霖形成于 一种同时具有上升气流与下沉气流的有组织 的积雨云中。在这种积雨云中, 强烈的上升气 流不仅给雹云输送了充分的水汽, 而且支撑着 冰雹粒子在云中的滞留。当冰雹颗粒生长到相 当大程度, 上升气流无法支撑冰雹重量时, 冰 雹颗粒就会从空中芏落, 最后在地面形成了降 雹。因此, 冰雹粒径的大小决定于积雨云中上 升气流大小和温度层结等气象条件。

在气象学中, 冰雨的严重程度, 有时也用 物体的大小来形容。例如, 小如绿豆、黄豆, 大似栗子、鸡蛋等形象化词语表示冰雱的形 态。除使用形态分类方法描述冰雱的严重度 外, 也可以用定量方法划分降䨖的严重度。大 致上, 气象学中将降雨分成软雨和冰雨。软雨 的尺寸是 2 5mm, 冰雹尺寸大于 $5 \mathrm{~mm}$ 。也有 根据冰䨌的直径分为三级: (1) 轻雨: 多数冰 雹直径不超过 $0.5 \mathrm{~cm}$; (2) 中雹: 多数冰雹直 径 $0.5 \sim 2.0 \mathrm{~cm}$; (3) 重雹: 多数冰雨直径 $2.0 \mathrm{~cm}$ 以上。

总之, 冰雨危害程度取决于冰霖尺度大 小、冰雱持续时间、冰雱发生频率、冰雱覆盖 区域面积等因素。

\section{1 .2 冰雹灾害的承灾体}

冰䨐的发生区域一般在地形复杂的北方 山区及丘陵地区。冰雹对承灾体的伤害与当地 的经济社会情况有密切的关系。在我国经济不 发达时期, 冰雹主要是对农业危害很大。猛烈 的冰雹常常毁坏庄稼、损坏房屋、人被砄伤、 打死牲畜等。现在, 随着我国经济的发展, 城 市公共设施和家庭汽车等成为了新的受灾体。 综合地分析, 我国冰雱灾害的主要危害表现在 农作物损失、人员伤亡、牲畜伤亡、汽车损失 和建筑物损失等方面。

\section{2 冰雨灾害的风险计算方法}

\subsection{1 单次冰雨过程的风险计算}

冰雹灾害的损失的形成, 主要取决于冰雨 的物理特征和风险单元的暴露性和脆弱性。依 靠这些主要的冰雹灾害的风险指标, 可以建立 下面的冰雹风险评估的数学模型:

$$
R_{1}=A^{*} f(E) * \sum_{i=1}^{n}\left(D_{i} * C_{i}\right)
$$

上式中, $\mathrm{A}$ 是冰雹的覆盖面积, $\mathrm{D}_{\mathrm{i}}$ 是第 $\mathrm{i}$ 种承 载体的密度, $\mathrm{C}_{\mathrm{i}}$ 是第 $\mathrm{i}$ 种承灾体的单位价值, $\mathrm{f}(\mathrm{E})$ 是依赖于冰雹降落动能的承灾体损失系 数。

\section{2 .2 年均冰雹风险估算}

在某地区，一年中可能发生多起冰雱灾 害, 但是冰雨灾害的发生次数可能是一个随机 的过程。那么, 一年的冰䨌损失风险可以用 下式估算:

$R_{a}=N_{\max } * p(N<n) * R_{1}$

式中, $\mathrm{R}_{\mathrm{a}}$ 是地区年均冰需损失, $\mathrm{N}_{\max }$ 是某一地 区的最大降雨日数, $\mathrm{P}(\mathrm{N}<\mathrm{n})$ 是小于 $\mathrm{n}$ 天的 降䨌累积概率, $\mathrm{R}_{1}$ 是单次冰䨌过程的总损失, 可以利用公式 (1) 计算。式中 $N_{\text {max }}$ 和 $P(N<n)$ 相乘的结果表示了统计意义上的小于 $\mathrm{n}$ 天的 降雨日数的平均值。

\section{3. 冰雹灾害承灾体因子的量化方案}

暴露量的评估就是定量地估算孕灾环境 中的承灾体暴露程度。在不同的条件下, 冰需 致灾因子对承灾体的危害是有差异的。如果用 概率风险评估承灾体的暴露量, 则暴露量的概 率是一个条件概率, 即承灾体完全或部分暴露 在致灾因子损害范围中的发生概率。

\section{1 冰霄灾害发生日数的计算}

估算冰䨨灾害发生日数的目的是在给定 的时间 $\mathrm{t}$ 内估计冰䨌事件的发生概率。一般情 况下, 人为或自然现象产生的灾害事件的发生 次数被认为服从泊松概率。因此, 冰雱日数的 出现概率可以利用 Poisson 分布模型来模拟。 在任意给定时间间隔 $\mathrm{t}$ 发生的事件数 $\mathrm{N}(\mathrm{u})$ 的泊松分布为:

$$
P[N(u)=n \mid \lambda(u), t]=\frac{1}{n !}(\lambda(u), t)^{n} e^{-\lambda(u) t}
$$

式中事件发生次数为 $n, \lambda(u)$ 是指阈值超过 $u$ 的泊松参数。对于单位时间 (如一年) 中的泊 松分布可以简化为下式:

$$
P[N(u)=n \mid \lambda(u)]=\frac{\lambda(u)^{n}}{n !} e^{-\lambda(u)}
$$

在一定周期中, 多次发生的冰雹灾害事件 可以使用累积分布进行估算。一年中, 发生次 数小于 $\mathrm{n}$ 次的冰雨发生概率总和可以使用下 面的累积分布计算: 
$P[N(u)<n]=\sum_{i=1}^{n} \frac{\lambda(u)^{i}}{i !} e^{-\lambda(u)}$

\section{2 冰雹灾害的暴露量的易损价值估算函数}

评估冰雹灾害的风险, 首先要估算冰雱灾 害的承灾体的经济价值。尤其是冰霄影响区域 的承灾体的易损价值评估。

某一个区域中的承载体的易损价值取决 于承灾体的数量和承灾体价值的密度。由于区 域中承灾体的数量和承灾体的价值分布是一 个随机性很大的事件, 同时这些承灾的信息在 现实世界中有具有不完备信息的特征。这中不 完备信息特征为灾害损失分析带来了极大的 困难。但是, 这些信息通常又服从于某种统计 分布函数, 而且这种分布函数的均值和方差是 较好获取的信息。这样就可以利用承灾体的概 率分布函数可以解决它的信息不完备性的缺 陷。

因此, 利用承灾体的价值分布函数可以较 好地估算承灾体暴露量的易损价值。如果某一 地区的承灾体的价值分布符合正态正态分布 规律, 则这个区域的承灾体暴露量的易损价值 就可以表示为:

$$
C=\int_{x_{0}}^{x} \frac{M x}{\sigma \sqrt{2 \pi}} e^{-\frac{1}{2}\left(\frac{x-\mu}{\sigma}\right)^{2}} d x
$$

在上式中, $\mathrm{x}$ 是承担风险的承灾体的数量变 量, 估算方法为 $x=A * D_{i}, A$ 是冰霄致灾因子 的覆盖面积, $D_{i}$ 是某区域中第 $\mathrm{i}$ 种承载体的数 量密度。 $\sigma$ 是承灾体数量的方差, $\mu$ 是承灾体的 数量均值, $\mathrm{M}$ 是单个承载体的价值均值。利用 这种方法, 可以将有限的承灾体易损值信息扩 展为较详细的承灾体易损值信息。

\section{3 冰雹造成的经济损失率的计算函数}

冰雹袭击承灾体后, 并非承灾体的全部价 值丧失, 有时只是部分价值丧失。这样, 为了 有效地评估冰雹灾害的经济损失, 就要建立冰 雨灾害的经济损失函数。

冰雨降落对承灾体的损害程度取决于冰 雹的降落动能。因为, 冰雹较小时, 降落动能 小, 对承灾体的损害的可能性小; 冰雹较大时, 降落动能大, 对承灾体的损害可能性大。这样, 可以利用冰雨动能的特性, 在冰雨灾害承灾体 的损失率和冰雹动能之间建立一种函数关系。 冰䨌承灾体的经济损失率可以采用 $\mathrm{S}$ 型函数 表示:

$$
f(E)=\left\{\begin{array}{l}
0 \ldots \ldots \ldots \ldots \ldots \ldots \ldots \ldots \\
\frac{1.0-e^{-b(E-a)}}{1.0+e^{-b(E-a)}} \ldots E>a
\end{array}\right.
$$

公式中, $\mathrm{E}$ 是冰雹的降落动能。 $\mathrm{a} 、 \mathrm{~b}$ 是 $\mathrm{S}$ 型的 调节参数, $\mathrm{a}$ 代表了冰雱对承灾体损害的最小 动能, $\mathrm{b}$ 是与降雹直径相关的调节参数, 它可 以根据实际的统计资料将损失率函数调节到 符合地区的冰雹灾害的损失率。

单个冰雹的降落动能可以用动能公式 $E=\frac{1}{2} m u^{2}$ 计算。在冰雱的动能计算公式中, 一般情况将冰雹颗粒假设为球形。这样，冰雨 颗粒的质量为:

$$
m=\frac{\rho_{i} \pi}{12} D^{3}
$$

式中, $\mathrm{D}$ 是冰䨠的直径, $\rho_{i}$ 是冰雹的密度 (取 $\left.846 \mathrm{~kg} / \mathrm{m}^{3}\right)$ 。

在冰雹动能计算中的另一个变量是冰雹 的速度。冰䨌由于受到空气的阻力作用, 在快 要降落到地面的一段时间内, 冰雹颗粒是处于 近似匀速运动。这时的冰雹运动速度就是冰雹 的降落末速度。冰雹降落末速度公式计算如 下:

$$
V=\left(\frac{4 \rho_{i} g D}{3 \rho_{a} C_{D}}\right)^{\frac{1}{2}}
$$

式中, $\rho_{a}$ 是空气密度 $\left(\right.$ 取 $\left.0.9 \mathrm{~kg} / \mathrm{m}^{3}\right), C_{\mathrm{D}}$ 是阻 力系数 (取 0.5 )。

综合冰雱颗粒的质量公式和降落末速度 公式, 单个冰雹的降落动能可以写成下式:

$$
E=\frac{\rho^{2}{ }_{i} g \pi}{9 \rho_{a} C_{D}} D^{4}
$$

但是在一次冰雹过程中, 降落的冰雹颗粒 数量很大, 而且冰雹的直径大小是不相等的。 在冰雹的损失评估中, 可以用平均动能计算损 失效率。假设冰雹的直径服从正态分布, 则冰 雹的平均动能为:

$$
\bar{E}=\int_{D_{1}}^{D_{2}} \frac{\rho^{2}{ }_{i} g \pi D^{4}}{9 \rho_{a} C_{D} \sigma \sqrt{2 \pi}} e^{-\frac{1}{2}\left(\frac{D-\mu}{\sigma}\right)^{2}} d D
$$

式中 $\mathrm{D}_{1}$ 和 $\mathrm{D}_{2}$ 是某次冰䨌天气过程中, 可以成 灾的冰雹颗粒的最小直径和最大直径。

\section{4. 冰霨灾害的承灾体的损失函数的参数确定}

冰霨对承灾体的损害程度取决于冰雨的 降落动能和承灾体的材质。一般情况下, 承灾 体的材质有钢材、玻璃和农作物等。例如, 冰 雹灾害对汽车的直接破坏主要是车身钢板壳 的凹陷或者挡风玻璃的破碎。因此, 对于汽车 承灾体造成的直接经济损失也就是车身钢板 外壳和玻璃的置换费用。 
在实际的冰䨙灾害的损失评估工作中, 冰 䨌造成的各种经济损失的实际数据不易收集, 以及冰雹灾害数据的明显随机特性使得小样 本数据的可靠性低。为了能在评估工作中弥补 这种信息中的不足, 本节利用 ANSYS 软件模 拟各种粒径的冰雱以不同的末速度撞击钢板 和玻璃, 通过模拟得到钢板和玻璃的应力场分 布、塑性应变场分布以及变形等多种结果, 从 而确定冰雹对钢板和玻璃的损伤。

通过数值模拟不同条件下冰雱对承灾体 的损伤程度, 进一步确定冰雹灾害对承灾体造 成的经济损失率函数中的 $\mathrm{a}$ 和 $\mathrm{b}$ 参数。

\subsection{ANSYS 的应力分析原理}

\subsection{1 冰雹撞击玻璃材料的非线性分析原理}

在 ANSYS 程序中, 分析冰雨对玻璃材料 的伤害效应时, 采用了非线性材料的结构分析 方法, 即牛顿一拉普森迭代法。牛顿一拉普森 迭代法通常有三种方法: 纯牛顿一拉普森迭代 法、修正牛顿一拉普森迭代法和准牛顿一拉普 森迭代法。前两者也称为切线刚度法, 而准牛 顿一拉普森迭代法也称为割线刚度法。在 ANSYS 程序中, 割线刚度法被称为弧长法。

在模拟计算中, 冰雹对玻璃撞击所造成的 损害主要取决于撞击的冲量。这个冲量一般包 括两部分: 冰䨌降落产生的坚直冲量和冰䨌相 对承灾体的水平冲量。

根据冰雱降落到地面时的末速度计算公 式, 将冰雹的各个物理常量代入公式, 可以等 到简化后的计算公式:

$$
V_{\perp}=\left(\frac{4 \rho_{i} g D}{3 \rho_{a} C_{D}}\right)^{\frac{1}{2}} \approx 156.7 \sqrt{D}
$$

式中, $V_{\perp}$ 是冰霄末速度 $(\mathrm{m} / \mathrm{s}), \mathrm{D}$ 是冰雱的 直径 $(m)$ 。 为:

同样, 冰䨌的质量 (千克) 也可以简化

$$
m=\frac{\rho_{i} \pi}{12} D^{3} \approx 221.5 D^{3}
$$

这样, 冰雹降落产生的坚直冲量就可以简 化为只与冰雹直径有关的函数关系:

$$
I_{\perp}=m V_{\perp}=\frac{\rho_{i} \pi}{12} D^{3}\left(\frac{4 \rho_{i} g D}{3 \rho_{a} C_{D}}\right)^{\frac{1}{2}} \approx 34714 D^{\frac{7}{2}}
$$

根据防雱试验的结果, 水平风速对于直径 小的冰雹影响较大, 然而对于直径大于 $3 \mathrm{~cm}$ 冰 雱的影响可以忽略。在分析冰雱的坚直冲量 时, 直径小于 $3 \mathrm{~cm}$ 的冰䨙的实际降落末速度 可以使用静止大气中冰雹下落的末速度乘以 修订系数 1.22 。

对于冰霖的水平冲量, 可以使用下式计 算:

$$
I_{H}=m V_{H}=\frac{\rho_{i} \pi}{12} D^{3} V_{H} \approx 221.5 D^{3} V_{H}
$$

式中的 $\mathrm{V}_{\mathrm{H}}$ 是冰雹的水平运动分量。

4.1.2 冰雹撞击玻璃的模拟结果分析

在实际情况中, 如果承灾体为汽车时, 并 假设汽车挡风玻璃的倾角为 $\alpha$ 。则冰雹撞击挡 风玻璃的合冲量为:

$$
I=I_{\perp} \cos \alpha+I_{H} \sin \alpha
$$

汽车设计中的挡风玻璃倾角 $\alpha$ 一般为 $30^{\circ} \sim 45^{\circ}$, 而且现在轿车挡风玻璃倾角设计得 越来越小, 有些已经达到 $20^{\circ}$ 。为了讨论问题 的方便，本文后面计算中的倾角 $\alpha$ 取 $30^{\circ}$, 则 冰雹撞击汽车挡风玻璃的合冲量:

$$
I=I_{\perp} \cos \alpha+I_{H} \sin \alpha=0.866 I_{\perp}+0.5 I_{H}
$$

冰雹对玻璃的集中载荷 $\mathrm{F}$ 可以近似通过 冲量与冲力的关系得到, 可以表示为: $F=I / \Delta t$ 。根据以往的试验结果, $\Delta t$ 一般取 $0.001 \mathrm{~s}$ 。

当冰雹粒径达到一定直径大小时, 冰雹对 玻璃的集中载荷 $\mathrm{F}$ 使得玻璃的形变会变得很 大。当应力达到一定阈值时, 会因为应力超过 了玻璃的抗压强度, 造成玻璃破碎。

使用 ANSYS 的模拟软件，对于 $0.5 \sim 10.0 \mathrm{~cm}$ 的冰雱撞击普通玻璃和钢化玻璃 进行模拟（见图 1)。模拟结果表明（表 1), 当冰雱直径达到 $4 \mathrm{~cm}$ 时, 钢化玻璃被撞击后 产生的形变约为 $1 \mathrm{~mm}$, 可认为此时玻璃产生 裂纹, 因此, 冰雹对钢化玻璃造成破坏的最小 粒径为 $4 \mathrm{~cm}$ 。

对于普通玻璃, 冰䨨直径达到 $2 \mathrm{~cm}$ 时, 玻璃的形变已经大于 $1 \mathrm{~mm}$, 超出了钢化玻璃 的形变量。可以认为 $2 \mathrm{~cm}$ 的冰雹会造成普通 玻璃破碎。

表 1 不同粒径冰雹撞击不同速度车辆造成挡风玻璃的形变量

\begin{tabular}{|l|l|l|l|l|l|l|l|}
\hline 冰雨粒径 $(\mathrm{cm})$ & 0.5 & 1.0 & 2.0 & 3.0 & 4.0 & 6.0 & 10.0 \\
\hline 普通玻璃 $(\mathrm{mm})$ & 0.011 & 0.111 & 1.151 & 4.564 & 12.228 & 81.059 & - \\
\hline 钢化玻璃 $(\mathrm{mm})$ & 0 & 0.009 & 0.09 & 0.365 & 0.967 & 5.068 & 21.953 \\
\hline
\end{tabular}




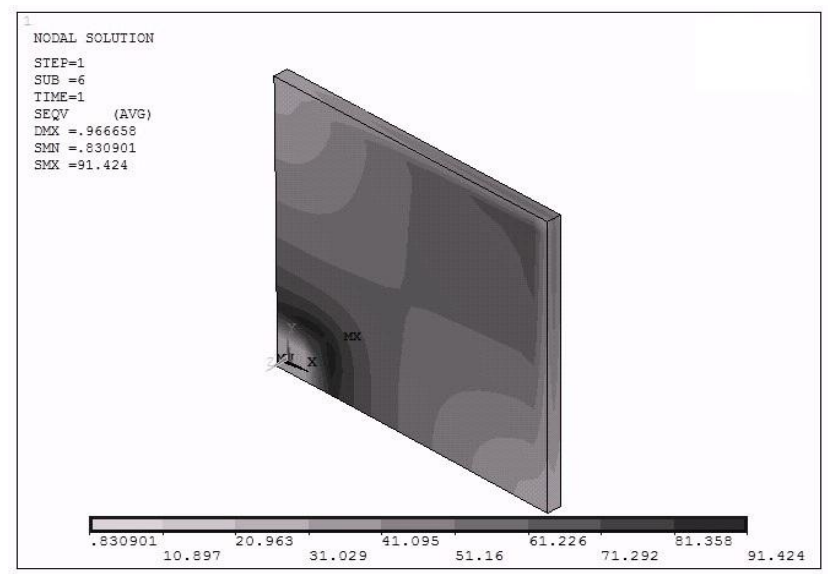

图 1 直径 $4 \mathrm{~cm}$ 冰雱撞击挡风玻璃（钢化玻璃）模拟结果

其中: $\mathrm{DMX}$ 为最大形变 (单位: $\mathrm{mm}$ ); $\mathrm{SMX}$ 为最大应力 (单位: $\mathrm{MPa}$ )

\section{2 冰雹撞击钢板的模拟原理和分析}

\subsection{1 冰䨌撞击钢板的显式非线性分析}

在 ANSYS 程序中，对于钢板的模拟不同 于玻璃的模拟方法。钢板的模拟采用了 LS-DYNA 的方法。LS-DYNA 是一个以显式 为主，兼顾隐式的非线性动力有限元分析程 序。它主要是在 $t+\Delta t$ 时计算位移和平均加速 度:

$$
\left\{u_{t+\Delta t}\right\}=[K]^{-1}\left\{F_{t+\Delta t}^{a}\right\}
$$

对于线性问题，由于 $\mathrm{K}$ 是线性的，解是 无条件稳定，计算过程可以采用大的时间步。 对于非线性问题，通过一系列线性逼近 (牛顿 -拉普森迭代法) 来获取解, 要求转置非线性 刚度矩阵 K, 收敛需要小的时间步。

LS-DYNA 的显式时间积分采用中心差分 法在时间 $\mathrm{t}$ 求加速度:

$$
\left\{a_{t}\right\}=[M]^{-1}\left(\left[F_{t}^{e x t}\right]-\left[F_{t}^{\text {int }}\right]\right)
$$

式中 $\mathrm{F}_{\mathrm{t}}^{\mathrm{ext}}$ —施加外力和体力矢量;

$\mathrm{F}_{\mathrm{t}}^{\mathrm{int}}$ 一内力矢量, 它由下式构成:

$$
F_{t}^{\mathrm{int}}=\sum\left(\int_{\Omega} B^{T} \sigma_{n} d \Omega+f^{h g}\right)+F^{\text {contact }}
$$

式中三项依次为当前时刻单元应力场等效节
点力、沙漏阻力 (为克服单点高斯积分引起的 沙漏问题而引入的粘性阻力）和接触力矢量。 4.2.2 冰䨌撞击钢板的模拟试验

冰䨌对钢板材料的承灾体撞击时, 会对钢 板材料的承灾体造成损坏。然而，这种损坏的 程度与冰雹的特性冰 (雹的直径、质量、撞击 的速度) 和被撞击物的特性 (几何特性和材料 的特性) 密切相关。由于真实的损失数据很难 获取, 同时在实际操作中又存在着各种各样的 困难, 因此冰雹撞击的数值模拟就非常必要。 通过数值模拟实验可以重现撞击过程, 分析撞 击结果。

本节对钢板材料的实验模拟, 材料参数以 冰雹的承灾体汽车为对象。实验目的是利用 ANSYS/LS-DYNA 软件, 通过计算不同直径冰 雹的降落动能, 确定不同冰雹动能撞击汽车钢 板的损伤程度。同样，利用公式 (16)，可以 确定冰雱撞击汽车外壳钢板末速度的计算公 式 $V=0.886 V_{\perp}+0.5 V_{H}$ 。

一般来说, 汽车外壳钢板的厚度为 0.6 0.8mm, 因此, 本文模拟中的钢板厚度取 $0.8 \mathrm{~mm}$ 。根据模拟结果, 随着冰雹粒径的增大, 冰雹颗粒对钢板的撞击形变也增大 (见图 2)。 当冰雹颗粒直径为 $3.0 \mathrm{~cm}$ 时, 钢板形变为 $3 \mathrm{~mm}$

(见表 2)。这种变形已对车辆表面造成伤害, 可以认为冰雨对钢板造成破坏的最小粒径为 $3.0 \mathrm{~cm}$ 。

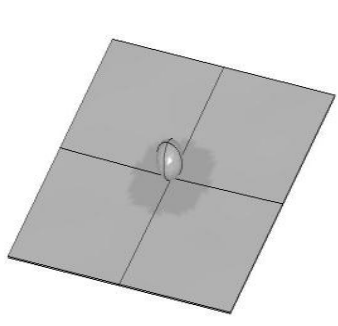

(a)
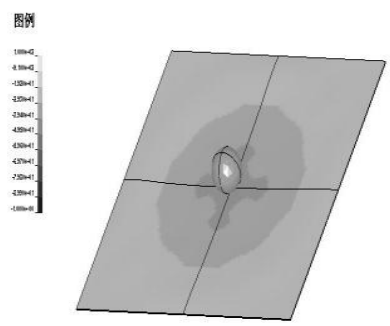

(b)
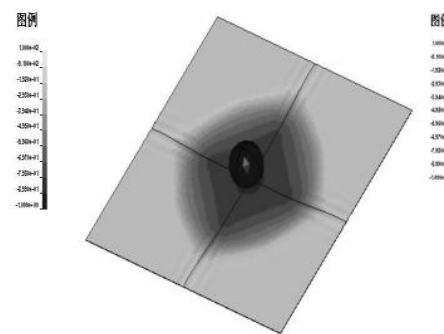

(c)

图 2 直径 $2.0 \mathrm{~cm}(\mathrm{a}) 、 3.0 \mathrm{~cm}(\mathrm{~b})$ 和 $6.0 \mathrm{~cm}(\mathrm{c})$ 的冰雹撞击钢板的模拟结果 
表 2 不同粒径冰雨撞击钢板的形变量

\begin{tabular}{|l|c|c|c|c|c|c|c|}
\hline 冰需粒径 $(\mathrm{cm})$ & 0.5 & 1.0 & 2.0 & 3.0 & 4.0 & 6.0 & 10.0 \\
\hline 钢板型变量 $(\mathrm{cm})$ & 0 & 0.036 & 0.13 & 0.30 & 0.51 & 1.05 & 28 \\
\hline
\end{tabular}

\section{3 汽车的总损失系数的确定}

公式 (7) 的损失率函数是估算冰雹灾害 损失的关键性因素。如果确定了冰雹伤害承灾 体的最小直径, 就可以确定公式 (7) 中的冰 雹最小动能参数 $\mathrm{a}$, 然而参数 $\mathrm{b}$ 可以利用已有 的数据来确定, 使得损失函数接近于真实的损 失情况。

现代化城市中的大量汽车的出现, 增加了 城市的冰雹灾害承灾体的类型。冰雹对汽车造 成的损害主要包括车身钢板外壳的变形和玻 璃破裂。因此, 对于汽车损失的评估分成了钢 板表面破坏和玻璃的破碎两部分。因此, 损失 率函数也分成了车身钢板表面损失和玻璃的 损失。

为了讨论汽车损失率问题的方便, 分别用 下标 $\mathrm{s}$ 和 $\mathrm{g}$ 表示钢板和玻璃。对于挡风玻璃来 说, 参数 $\mathrm{a}_{\mathrm{g}}$ 取直径为 $4 \mathrm{~cm}$ 的冰䨌的降落动能, 则小于 $4 \mathrm{~cm}$ 的冰䨌对挡风玻璃造成的损失为 零, 即 $f(E)_{g}=0$ 。而对于车身钢板来说, 参数 $a_{s}$ 取直径为 $3.0 \mathrm{~cm}$ 的冰雹的降落动能, 则小于 $3.0 \mathrm{~cm}$ 的冰雹对汽车挡风玻璃造成的损失为 零, 即 $f(E)_{s}=0$ 。

由于参数 $\mathrm{b}$ 的确定与地区的经济发展水 平有密切关系。这里, 作者根据天津市的历史 统计数据, 确定公式 (7) 中适合天津市冰雹 灾害对车辆挡风玻璃和车身钢板损失率函数 的系数 $b_{\mathrm{g}}$ 和 $\mathrm{b}_{\mathrm{s}}$, 这两个值分别值 $0.01 \mathrm{D}_{\mathrm{g}}$ 和 $0.01 \mathrm{D}_{\mathrm{s}}$, 其中, $\mathrm{D}_{\mathrm{g}}$ 和 $\mathrm{D}_{\mathrm{s}}$ 分别是挡风玻璃和汽 车钢板的形变量, 直径单位为毫米。这样, 冰 雱对汽车造成的损害的总损失系数 $f(E)=f(E)_{g}+f(E)_{s}$ 。

\section{4 冰雹造成农业损失系数的确定}

冰雹对农业生产地区造成的危害主要是 农业作物, 以及生产农作物的农业设施（如蔬 菜大棚的塑料薄膜和玻璃)。冰雨出现时常与 短时大风和强降雨同时出现, 对生长中的农 作物破坏极大。

农作物的枝叶、茎秆、果实受到冰雱颗粒 的打击, 会因叶子损失、茎秆折断、果粒脱落 等伤害而形成减产。苗期的农作物遭受冰雹袭
击后, 可使幼苗受伤而不能正常生长, 若幼苗 被砸伤过重, 则需重新播种而延误农事季节。 农作物在灌浆成熟期受到冰雱袭击, 会直接 影响并阻碍正常灌浆成熟而造成严重减产和 品质变劣。果树在开花座果时遭受冰雹灾害, 会形成严重的落花落果现象而导致大幅度减 产。虽然有的幼果被冰雱打伤后也可以发育成 熟, 但是带有雱伤的果实的商品价值会大幅 度降低。然而, 果实在成熟期遭受雱灾后, 果 实容易腐烂, 也不耐财存, 常带来无法弥补的 经济损失。

冰雹的危害程度主要取决于冰雹的强度、 密度、持续时间和降雹的季节。冰雹直径越大, 密度越大, 危害也越严重, 成灾概率越大。

根据以往研究, 当冰雹直径大于 $0.5 \mathrm{~cm}$ 时，就会对农业造成损失。因此，对于农作物 的雹灾风险, 公式 (7) 中的调节参数 a 就可 以取 $0.5 \mathrm{~cm}$ 冰雨的动能。根据天津市冰雨灾害 对农业造成损失的历史统计数据, 公式 (7) 中的系数 $\mathrm{b}$ 可以取值 0.01 。

\section{5. 冰電灾害的损失风险评估分析}

由于冰雹灾害具有明显的地区性特点, 本 节将以天津地区为冰雹灾害的孕灾环境, 使用 冰雹灾害评估模型对天津市冰雹灾害的损失 风险进行评估分析。根据天津市的实际情况, 冰雹灾害的承灾体主要为汽车和农作物。

\section{1 风险评估中的数据说明}

冰雹的发生天数的数据来自天津市气象 部门 1958 年至 2003 年的观测统计数据 (见表 3 )。冰雹直径数据也是来自气象部门的观测数 据。

轿车统计数量和农业产值来自天津市 2007 年的统计年鉴数据（见表 4 和表 5)。因 为统计年鉴中只将轿车分成微型汽车、小型汽 车和中型汽车三类。为了讨论汽车价值的需 要, 分别将这三类车作为低价、中价和高价车。

天津市各个区县的农作物的价值, 依据统 计年鉴中的农业总产值计算。

表 31958 年至 2003 年天津市及郊区的总降雨频率

\begin{tabular}{|c|c|c|c|c|c|c|c|}
\hline 年次数 & 0 & 1 & 2 & 3 & 4 & 5 & 合计 \\
\hline 总统计数 & 79 & 53 & 32 & 18 & 1 & 1 & 184 \\
\hline 百分比 & 42.92 & 28.81 & 17.39 & 9.78 & 0.55 & 0.55 & 100.00 \\
\hline
\end{tabular}




\begin{tabular}{|l|l|l|l|}
\hline 车类型 & 微型 & 小型 & 中型 \\
\hline 数量 & 102784 & 504198 & 26002 \\
\hline
\end{tabular}

表 5 各区县 2006 年农业总产值（万元）

\begin{tabular}{|l|l|l|l|l|l|l|l|l|}
\hline 东丽区 & 西青区 & 津南区 & 北辰区 & 武清区 & 宝坻区 & 宁河县 & 静海县 & 蓟 县 \\
\hline 43939 & 64145 & 22252 & 52169 & 274835 & 167013 & 126997 & 126366 & 178131 \\
\hline
\end{tabular}

\section{2 冰䨌评估模型的模拟分析}

\subsection{1 冰霞天气的情景设计}

由于某一地区的冰雹发生天数, 以及冰 雹的粒径都是一个随机变化的数据。因此, 在 风险评估中要考虑这些随机特性。

一般情况下, 冰雹的发生天数服从泊松 分布。这样, 依照泊松分布规律, 利用天津市 的冰雹发生日数的历史统计数据, 可以确定天 津地区的冰雹发生日的泊松分布函数的 $\lambda$ 参数 为 0.978 。而且, 表 3 中的历史观测数据表明 天津地区的最大年将雹日数是 5 天。
如果在冰雹灾害风险评估中, 假设冰雹 的直径服从正态分布规律。这样就可以建立了 天津地区的冰雹直径的正态分布模型。通过分 析天津的历史降雹的粒径数据, 冰雹灾害的平 均冰䨌颗粒的直径约 $2.5 \mathrm{~cm}$, 方差约 3.0 ,

以冰䨌发生天数的泊松分布函数和冰霄 直径的正态分布模型，结合模特卡罗随机模拟 方法, 生成天津地区冰雹天气的 10 个情景 (表 6)。这些以天津市历史统计资料为基础生成的 冰雹天气情景, 可以作为分析天津是冰雹灾害 风险的致灾因子样本。

表 6 蒙特卡罗模拟的冰雱天气情景

\begin{tabular}{|l|l|l|l|}
\hline 序号 & 冰雹日数 & $\mathrm{P}(\mathrm{N}<\mathrm{n})$ & 雹径 $(\mathrm{cm})$ \\
\hline 1 & 2 & 0.548 & 4.33 \\
\hline 2 & 1 & 0.368 & 3.18 \\
\hline 3 & 1 & 0.368 & 5.50 \\
\hline 4 & 1 & 0.368 & 6.15 \\
\hline 5 & 2 & 0.548 & 0.87 \\
\hline 6 & 2 & 0.548 & 5.24 \\
\hline 7 & 1 & 0.368 & 1.98 \\
\hline 8 & 2 & 0.548 & 1.50 \\
\hline 9 & 2 & 0.548 & 4.12 \\
\hline 10 & 1 & 0.368 & 5.30 \\
\hline 平均 & 1.5 & $/$ & 4.12 \\
\hline
\end{tabular}

\subsection{2 冰䨌天气的汽车损失情况评估}

根据天津市统计年鉴资料, 估算天津市 轿车的价值均值约 7.58 万元, 方差 4.74。使 用天津市的 10 个冰雹灾害的天气情景, 并采 用蒙特卡罗模拟的方法产生受损的汽车数量, 通过轿车价值的正态分布模型估算出受损汽 车的总价值 (表 7)。

随机模拟结果显示, 受灾区域的汽车损 失情况与车辆的平均价值关系密切。在受灾车 辆数变化不大的情况下, 车辆平均价值越大, 冰雹灾害的损失越大。

同时, 模拟结果也显示出冰雹直径的变 化对损失率的影响。当冰雹冰霄直径大于对承 灾体伤害直径时, 相应的损失率函数就为大于 0 的正数。如果冰雹的直径小于对承灾体伤害 直径时, 相应的损失率函数就为 0 。而且冰雹 直径越大, 车辆本身的损失率值也越大。
可以看出, 冰雹灾害的损失评估模型反映了承 载体的价值和致灾因子的强度等综合因素的 作用。

\subsection{3 农作物损失情况评估}

对于农业损失情况评估，使用了天津市 的 9 个具有农业生产的区县的统计数据（表 5 )。并且假设这 9 个区县中的农作物的产值的 面积密度分布为均匀分布。冰霄灾害的影响区 域, 以 $6 \mathrm{~km}$ 宽度为基准, 长度是冰霖灾害经 过区县的路径长度。

冰霖天气数据仍然使用表 6 的 10 个冰雹 天气情景数据。估算的天津地区各个农业生产 区的一年内的冰雹灾害造成的损失情况如表 8 所示。

依据表 8 的评估结果和表 6 的冰霄天气情 景数据可以看出, 冰雹灾害评估模式评估的农 作物的经济损失是随着冰雱的粒径增加, 冰雹 
灾害的损失也在增加。而且冰雹天数多的情景 造成的农业损失明显高于发生天数小的情景。

表 7 冰雹天气造成的汽车损失（万元）

\begin{tabular}{|l|r|l|l|l|l|r|}
\hline 序号 & $\mathrm{f}(\mathrm{E})_{\mathrm{g}}$ & $\mathrm{f}(\mathrm{E})_{\mathrm{s}}$ & $\mathrm{f}(\mathrm{E})$ & 受损车辆数 & 受灾车辆均价 & 受灾车辆总损失 \\
\hline 1 & $2.858 \mathrm{E}-3$ & $4.416 \mathrm{E}-3$ & $7.274 \mathrm{E}-3$ & 317 & 7.58 & 9.5780 \\
\hline 2 & 0 & $1.910 \mathrm{E}-4$ & $1.910 \mathrm{E}-4$ & 454 & 6.07 & 0.1936 \\
\hline 3 & $6.017 \mathrm{E}-2$ & $1.996 \mathrm{E}-2$ & $8.013 \mathrm{E}-2$ & 748 & 12.77 & 281.6772 \\
\hline 4 & $1.594 \mathrm{E}-1$ & $4.036 \mathrm{E}-2$ & $1.997 \mathrm{E}-1$ & 366 & 0 & 442.7544 \\
\hline 5 & 0 & 0 & 0 & 0 & 11.83 & 0 \\
\hline 6 & $3.629 \mathrm{e}-2$ & $1.574 \mathrm{E}-2$ & $5.203 \mathrm{E}-2$ & 436 & 0 & 0 \\
\hline 7 & 0 & 0 & 0 & 0 & 0 & 0 \\
\hline 8 & 0 & 0 & 0 & 0 & 7.77 & 9.0851 \\
\hline 9 & $9.350 \mathrm{E}-4$ & $2.930 \mathrm{E}-3$ & $3.865 \mathrm{E}-3$ & 552 & 10.79 & 70.7043 \\
\hline 10 & $4.087 \mathrm{E}-2$ & $1.676 \mathrm{E}-2$ & $5.763 \mathrm{E}-2$ & 309 & 8.431 & 96.1 \\
\hline 平均 & - & - & - & 359.3 & & 0581 \\
\hline
\end{tabular}

表 8 冰䨚天气造成的农业损失（万元）

\begin{tabular}{|l|l|l|l|l|l|l|l|l|l|l|}
\hline 区县 & 1 & 2 & 3 & 4 & 5 & 6 & 7 & 8 & 9 & 10 \\
\hline 东丽区 & 4590.4 & 449.43 & 3943.7 & 5992.8 & 6.6865 & 9739.8 & 2680.3 & 65.503 & 3766.2 & 3418.2 \\
\hline 西青区 & 6701.3 & 656.11 & 5757.3 & 8748.7 & 9.7614 & 14219 & 3912.8 & 95.626 & 5498.1 & 4990.1 \\
\hline 津南区 & 2324.7 & 227.6 & 1997.2 & 3034.9 & 3.3863 & 4932.5 & 1357.4 & 33.173 & 1907.3 & 1731.1 \\
\hline 北辰区 & 5450.2 & 533.61 & 4682.4 & 7115.3 & 7.939 & 11564 & 3182.3 & 77.772 & 4471.6 & 4058.5 \\
\hline 武清区 & 28712 & 2811.1 & 24668 & 37485 & 41.824 & 60922 & 16765 & 409.72 & 23557 & 21381 \\
\hline 宝坻区 & 17448 & 1708.3 & 14990 & 22779 & 25.416 & 37021 & 10188 & 248.98 & 14315 & 12993 \\
\hline 宁河县 & 13268 & 1299 & 11399 & 17321 & 19.326 & 28151 & 7746.8 & 189.32 & 10885 & 9879.7 \\
\hline 静海县 & 13202 & 1292.5 & 11342 & 17235 & 19.23 & 28011 & 7708.3 & 188.38 & 10831 & 9830.6 \\
\hline 蓟 县 & 18610 & 1822 & 15988 & 24295 & 27.108 & 39486 & 10866 & 265.55 & 15268 & 13858 \\
\hline
\end{tabular}

\subsection{4 讨论}

模拟实验的结果说明通过概率方法和物 理方法结合构建的冰雨灾害的评估模型, 能够 综合反映致灾因子强度和承灾体价值情况。而 且, 评估模型可以应用于汽车、建筑玻璃和农 作物等损失评估。

但是, 评估模型中的承载体的价值损失率 函数的准确评估是一个复杂的问题, 同承灾体 的地理分布关系密切, 需要在实际工作中使用 实际数据不断地完善。

\section{6. 结论}

（1）针对冰雹灾害对社会经济造成的损 失风险问题, 作者利用物理方法和概率方法建 立了相应的冰雹灾害的风险评估的数学模型。

(2) 利用 ANSYS 软件和 LS-DYNA 软 件分别模拟了不同粒径的冰雹撞击汽车挡风 玻璃 (钢化玻璃) 和车身钢板以及建筑物玻璃 (普通玻璃) 造成的损害情况。模拟结果表明, 而当冰雹直径达到 $2.0 \mathrm{~cm}$ 时, 冰霄会对建筑物 的普通玻璃产生破坏。当冰霄颗粒直径为 $3.0 \mathrm{~cm}$ 时, 冰雹可以对汽车外壳钢板造成了损 失; 而当冰雹直径达到 $4.0 \mathrm{~cm}$ 时, 冰雹会对汽 车的挡风玻璃产生破坏。

（3）根据天津市的历史统计资料，利用 建立的冰雹灾害风险评估数学模型, 对天津市 的冰䨌灾害损失做了模拟分析。分析结果表
明, 在资料信息不足的情况下, 本数学模型是 有效的冰霄灾害损失的综合评估模型。

本研究只是对冰雹灾害风险评估建模的 理论研究, 如果将冰䨌风险模型与 GIS 系统相 结合, 可以做到冰雹灾害风险的定点风险分 析, 这会更加有利于冰雱灾害的风险管理。

\section{参考文献:}

1. S. A. Changnon, The scales of hail. Journal of Applied Meteorology , 16(1977) 626-648.

2. I. Kuhnel, The use of a multifactor Southern Oscillation Index for the estimation of annualhailstorm frequencies in the Sydney area, Int. J. Climatol,18(1998) 841-858.

3. W. Wang and H. Z.Jia, Forecasting Hail with Radar Vertically Integrated Liquid Data, Meteorological Monthly, ,28(1) (2002) 47 48.

4. Y. L. Jin and Q. Zhang, A Case Study of Synoptic Process and Inner Structure of Hails in Beijing, Meteorological Monthly,28(1) (2002)18 20.

5. A. Bengtsson and C. Nilsson, Extreme value modelling of storm damage in Swedish forests, Nat. Hazards Earth Syst. Sci., 7(2007) 515 521.

6. R. LEIGH and I. KUHNEL, HailstormLoss Modelling and Risk Assessment in the Sydney Region, Australia. Natural Hazard, 
24(2001) 171 185.

7. P. DAMIR, Comparison of Hail Characteristics in NW Croatia for Two Periods, Natural Hazards, 29(2003) 543 552.

8. S. Hergarten, Aspects of risk assessment in power-law distributed natural hazards, Nat. Hazards Earth Syst. Sci., 4 (2004) 309 313.

9. J. W. Han, H. M. Wang and L. Wu, The analysis and assessment on thunderstorm and hail disasters and the counter mea ures in InnerMongolia, Journal of Arid Land Resources and Environment, 23 (7) (2009)31 38.

10. A. C. Stanley, Increasing major hail losses in the U.S., Climatic Change, 96(2009)
161-166.

11. M. Julian, Damaging hail hits VW, weather,63 ( 2008),p218.

12. European Commission ( eds.), First Report on the Harmonisation of Risk Assessment Procedures, (DG Health \& Consumer Protection, Brussels, 2000).

13. R. Edmund, F. Peter and R. David, Estimating Injury and Loss of Life in Floods: A Deterministic Framework, Natural Hazard, 36(2005) 43-64.

14. Y. Zhang and Z. Lei, Static Characteristic and Impact Fracture Behavior of Automobile Glass. Journal of Mechanical Engineering, 45(2)(2009) 64 75. 